\title{
Femoral Hernia with Obstruction
}

National Cancer Institute

\section{Source}

National Cancer Institute. Femoral Hernia with Obstruction. NCI Thesaurus. Code C35209.

The protrusion of contents of the abdominal cavity, into the femoral canal, which results in obstruction of intestinal peristalsis. 